\title{
Adenocarcinoma de próstata gleason clínico 8-10: influencia pronóstica en la supervivencia libre de progresión bioquímica
}

\author{
Rioja Zuazu J, Zudaire Berbera JJ, Rincón Mayans A, Rosell Costa D, Robles Garcia JE, \\ Berian Polo JM.
}

Departamento de Urología, Clínica Universitaria, Universidad de Navarra.

Actas Urol Esp. 2008;32(8):792-798

\section{RESUMEN}

ADENOCARCINOMA DE PRÓSTATA GLEASON CLINICO 8-10: INFLUENCIA PRONOSTICA EN LA SUPERVIVENCIA LIBRE DE PROGRESIÓN BIOQUÍMICA

Objetivo: Analizar las características del grupo de pacientes con Adenocarcinoma de próstata Gleason clínico 8-10 en nuestra serie tratados mediante prostatectomía radical, e intentar averiguar que factores dentro del grupo influyen en la progresión y supervivencia libre de progresión.

Material y Métodos: De una serie de 781 pacientes con Cáncer de Próstata T1-T2, tratados con Prostatectomía Radical entre 1990-2004, estudiamos 108 con Gleason clínico 8-10. Mediana de PSA de 12 ng/ml, el 50\% era T2.

Se han estudiado las variables relacionadas con la progresión bioquímica y la supervivencia libre de progresión bioquimica, comparando el grupo Gleason 8-10 con el resto y analizando dentro del grupo Gleason 8-10 las variables relacionadas con la progresión y Supervivencia libre de progresión, buscando un modelo predictivo. Se han utilizado tablas de contingencia y regresión logística. Para el análisis de la supervivencia, Kaplan-Meyer, log-rank y modelos de Cox.

Resultados: En el estudio de progresión bioquímica de la totalidad de la serie (781 pacientes). El Gleason clínico 8-10 es un factor influyente en el estudio univariado (OR:2,61 IC 95\%: 1,7-4).

En el estudio de supervivencia libre de progresión (SLP) de la totalidad de la serie (781 pacientes) la SLP de los Gleason clínico 8-10 a 3 y 5 años es de $56 \pm 5 \%$ y $35 \pm 7 \%$, significativamente peor que la del resto del grupo ( $<<0,0001)$. El estudio Multivariado de los factores influyentes en la SLP incluye el Gleason 8-10 como factor de influencia independiente (OR:2,6 IC 95\%: 1,6-4,12) p=0,003, junto al estadio clínico (OR:1,81 IC95\%:1,182,78) p<0,006, el PSA (OR: 1,03 IC 95\%:1,025-1,046) p<0,0001y el lado de afectación de la biopsia (OR:1,5 IC 95\%:1,01-2,24) p=0,045.

Dentro del Grupo Gleason Clínico 8-10 los factores influyentes en la SLP son: el PSA (OR:1,02 IC 95\%:1,003-1,04), y el estadio patológico (OR:3,84 IC 95\%: 1,77-8,27). Los pacientes pT2 tienen una supervivencia significativamente mejor que los pT3 a 3 y 5 años $(80 \pm 6 \%$; $54 \pm 13 \%$ y $40 \pm 7 \% ; 27 \pm 7 \%)(\mathrm{p}<0,0001)$. El mejor punto de corte para PSA es $11 \mathrm{ng} / \mathrm{ml}$. Los pacientes con PSA $<1 \mathrm{lng} / \mathrm{ml}$ tienen una supervivencia a 3 y 5 años significativamente mejor que los $>11 \mathrm{ng} / \mathrm{ml}(74 \pm 7 \%, 30 \pm 22 \%$ y $40 \pm 7 \%, 26 \pm 7 \%)(\mathrm{p}<0,0001)$.

Conclusión: El gleason clínico 8-10 es un factor negativo de influencia independiente en la supervivencia libre de progresión, pero su pronóstico es mejor si presentan un PSA previo $<11 \mathrm{ng} / \mathrm{ml}$ y si el estadio patológico es pT2.

Palabras clave: Cáncer de próstata. Gleason 8-10. Supervivencia libre de progresión.

\section{ABSTRACT}

GLEASON SCORE 8-10 PROSTATIC ADENOCARCINOMA: PROGNOSTIC INFLUENCE IN THE BIOCHEMICAL PROGRESSION FREE SURVIVAL

Objective: Hereby, we analyze the characteristics of the clinical Gleason 8-10 group of patients with in our series diagnosed of Prostate Cancer and treated by means of radical prostatectomy, and we try to ascertain which are the influence factors within this group upon progression and progression free survival.

Material and Methods: From the global series of 781 patients with T1-T2 prostate cancer treated by means of radical prostatectomy between 1990 and 2004, we study 108 with a Gleason score on the biopsy of $8-10$. Median PSA was $12 \mathrm{ng} / \mathrm{ml}$ and $50 \%$ were T2.

Variables related to biochemical progression and progression free survival have been studied, comparing the group of Gleason 8-10 with the rest and analyzing, within the Gleason 8-10 group which are the related variables with progression and progression free survival, trying to find a predictive model. Contingency tables and logistic regression have been employed. For the survival analysis, Kaplan Meyer curves, log-rank and Cox models.

Results: Actual State: 62,7\% (490/781) are alive and free of biochemical progression, $24,8 \%(194 / 781)$ are alive with biochemical progression, 2,9\% $(23 / 781)$ are dead by cancer and $1,9 \%(15 / 781)$ are dead by other cause and 7,6\% (59/781) are lost.

Biochemical progression study of the whole series (781 patients) Clinical Gleason score 8-10 is a influence factor on the univariate study (OR:2,61 IC 95\%: 1,7-4).

In the progression free survival study (PFS) of the whole series (781 patients) the PFS in Clinical Gleason $8-10$ at 3 and 5 years is $56 \pm 5 \%$ y $35 \pm 7 \%$, significantly worse than the rest of the group ( $<<0,0001)$. In the multivariate study of the influence factors on the PFS includes Clinical Gleason Score 810 as an independent prognostic factor (OR:2,6 IC 95\%: 1,6-4,12) p=0,003, together with the clinical stage (OR:1,81 IC95\%:1,18-2,78) p<0,006, the PSA (OR: 1,03 IC 95\%:1,025-1,046) p<0,0001 and the side of tumor on the biopsy (OR: 1,5 IC 95\%:1,01-2,24) $\mathrm{p}=0,045$.

In the clinical Gleason score 8-10 group the influent factors on the PFS are. PSA (OR:1,02 IC 95\%:1,003-1,04) and pathological stage (OR:3,84 IC 95\%: 1,77-8,27). Patients with a pT2 have a significantly better survival than those pT3 at 3 and 5 years $(80 \pm 6 \% ; 54 \pm 13 \%$ y $40 \pm 7 \% ; 27 \pm 7 \%)(p<0,0001)$. The best cut point for the PSA is $11 \mathrm{ng} / \mathrm{ml}$. Patients with a PSA $<1 \mathrm{lng} / \mathrm{ml}$ have a 3 and 5 years survival better than those with $>11 \mathrm{ng} / \mathrm{ml}$ PSA $(74 \pm 7 \%$, $30 \pm 22 \%$ y $40 \pm 7 \%, 26 \pm 7 \%)(\mathrm{p}<0,0001)$.

Conclusions: Clinical Gleason Score $8-10$ is a negative independent prognostic factor on the progression free survival, but its prognosis is better if they present a PSA prior surgery lower than $11 \mathrm{ng} / \mathrm{ml}$ and the pathological stage is a pT2.

Keywords: Prostate cancer. Gleason 8-10. Progression-free survival. 
$\mathrm{E}^{1}$ cáncer de próstata es la neoplasia visceral que con mayor frecuencia se diagnóstica, así como la segunda causa de muerte por cáncer en USA ${ }^{1}$. La prostatectomía radical es un tratamiento efectivo en pacientes con cáncer de próstata clínicamente localizado $^{2,3}$, siendo uno de los procedimientos que con mayor frecuencia se realiza en $\mathrm{USA}^{4}$.

Los pacientes con un cáncer de próstata de alto riesgo; Gleason $>8$, PSA $>20 \mathrm{ng} / \mathrm{ml}$ y estadio clínico $>$ T2c considerados dentro de esta categoría, presentan una alta probabilidad de fracaso terapéutico y una peor supervivencia ${ }^{5,6}$. El tratamiento de estos pacientes no está del todo definido, no existiendo un consenso acerca de cual es la modalidad más optima. En la era pre PSA, los pacientes con tumores pobremente diferenciados presentaban tumores voluminosos y un estadio T3c, indicativos de extensión extraprostática. Existía un cierto temor a la hora de ofrecer Prostatectomía Radical como tratamiento local a estos pacientes, debido a la alta incidencia de lesiones ganglionares (70-100\%) recurrencia local y sistémica, así como una pobre supervivencia $^{7,8}$. Sin embargo, en la era PSA, la mayoría de los pacientes se diagnostican con un cáncer de próstata organoconfinado. Actualmente los pacientes con un Gleason en la biopsia > 8 que presentan estadios T1c-T2 pueden ser considerados para tratamiento bien con prostatectomía radical o radioterapia externa ${ }^{9,10}$.

Múltiples nomogramas se han desarrollado con el objetivo de mejorar y predecir el pronóstico de los pacientes antes y después de la cirugía11-14. Las opciones terapéuticas de los pacientes diagnosticados de cáncer de próstata se basan en el PSA, el grado de Gleason de la biopsia y el estadio clínico ${ }^{15}$. Cada factor pronóstico es insuficiente para predecir el riesgo de recurrencia y progresión ${ }^{13}$; el grado de Gleason es un buen predictor del resultado terapéutico $^{16}$, pero el grado de Gleason de la biopsia puede variar con respecto al Gleason patológico de la pieza de prostatectomía, debido a un muestreo insuficiente durante la misma durante la biopsia, ya que por definición es la suma de las dos poblaciones más frecuentes del cáncer ${ }^{17}$. La diferencia entre el grado de Gleason de la biopsia con respecto al grado de Gleason de la pieza quirúrgica oscila entre $36 \%$ y $60 \%{ }^{18-20}$, aunque en un trabajo más reciente un $39 \%$ de los pacientes con grado de Gleason $\geq 8$ presentaron un grado de Gleason $\leq 7$ en la pieza de prostatectomía $^{21}$. Por lo tanto, las limitaciones del grado de Gleason clínico a la hora de tomar decisiones deben ser consideradas.

No existen trabajos aleatorizados comparando las opciones terapéuticas en los pacientes con cáncer de próstata de alto grado. Múltiples trabajos han discutido los resultados de la prostatectomía radical en pacientes con un grado de Gleason en la pieza quirúrgica $\geq 8$. Sin embargo, existe muy poca información acerca de los resultados terapéuticos de los pacientes con un grado de Gleason en la biopsia $\geq 8$; es por ello que nosotros presentamos los resultados obtenidos por nuestro grupo en el tratamiento de los pacientes con un grado de Gleason $\geq 8$ en la biopsia tratados mediante prostatectomía radical.

Los objetivos que nos hemos planteado son: valorar la sensibilidad del Gleason de la biopsia (Gleason clínico) como herramienta diagnóstica, analizar las diferencias en la Progresión Bioquímica y en la Supervivencia Libre de Progresión bioquímica entre los pacientes con Gleason 2-7 y 8-10, y Analizar en el Grupo Gleason 8-10 los factores influyentes en Progresión Bioquímica y Supervivencia Libre de Progresión.

\section{MATERIAL Y MÉTODOS}

Hemos revisado de una serie de una única institución (CUN) 781 pacientes consecutivos diagnosticados de cáncer de próstata clínicamente localizado (estadios T1-T2) y tratados mediante prostatectomía radical entre 1990 y 2004. Aquellos pacientes con un seguimiento inferior a dos años, prostatectomía radical de rescate y que estuviesen en tratamiento adyuvante, fueron desechados del estudio.

El estudio preoperatorio consistió en una historia clínica, exploración física (que incluía tacto rectal), análisis de PSA y biopsia prostática. La estadificación clínica se completo con una tomografía computerizada o resonancia magnética, así como una gammagrafía ósea. Para la realización de estas pruebas se siguieron las directrices de las guías de la EAU, mientras que la RM se realizó prácticamente a todos los pacientes, excluyendo aquellos que tenían un TC realizado, desde el año 2002.

A todos los pacientes se les realizó una prostatectomía radical retropúbica, utilizando una modificación de la técnica descrita por Walsh ${ }^{22}$, con linfadenectomía ilio-obturatriz bilateral. Se realizó con- 
servación de cuello vesical así como preservación de bandeletas neuro-vasculares en casos selectos. Aquellos pacientes que tenían tratamiento neoadyuvante su duración no fue mayor de tres meses y se suspendió después de la cirugía. Todas las piezas de prostatectomía así como los ganglios linfáticos fueron examinados por el mismo especialista en anatomía patológica (A.P.) utilizando la técnica descrita por True ${ }^{23}$.

Las piezas de prostatectomía fueron pesadas, medidas, cubiertas con tinta y fijadas en formol al $10 \%$; se realizaron cortes de $2 \mathrm{~mm}$. En el apex distal y en la base de la próstata, las vesículas seminales se separaron de la pieza y se examinaron. La próstata se seccionó en intervalos de $3 \mathrm{~mm}$ desde el ápex hasta la base. Para la estadificación se utilizó el sistema TNM de la American Joint Committee y se utilizó la escala de Gleason para clasificar el grado histológico. El Grado de Gleason se calculó como la suma de la población primaria y secundaria. El volumen tumoral se expresó como el porcentaje de la estimación visual del cáncer ${ }^{24}$.

Se han analizado los datos clínicos y patológicos de estos pacientes, incluyendo grado de Gleason de la biopsia, edad, PSA, estadio clínico, Gleason patológico, estadio ganglionar, extensión extraprostática, invasión de las vesículas seminales y márgenes quirúrgicos afectos. Después de la Prostatectomía radical se han realizado determinaciones de PSA con intervalos de 3 y 6 meses. Se consideró progresión bioquímica si el PSA era mayor de 0,4 $\mathrm{ng} / \mathrm{ml}^{25}$.

Estudiamos 108 con Gleason clínico 8-10. El grupo tenia una media y mediana de PSA de 16,8 $\mathrm{ng} / \mathrm{ml} ; 12 \mathrm{ng} / \mathrm{ml}$ respectivamente. El 50\% presentaba un estadio clínico T2 y en el $61 \%$ de los casos la biopsia pre cirugía tenía afectación bilateral. En la Tabla 1 se comparan las variables de este grupo con el resto de la serie.

Estadísticos: Se han estudiado las variables relacionadas con la progresión bioquímica y la supervivencia libre de progresión bioquímica, comparando el grupo Gleason 8-10 con el resto y analizando dentro del grupo Gleason 8-10 las variables relacionadas con la progresión y Supervivencia libre de progresión, buscando un modelo predictivo. Se han utilizado tablas de contingencia y regresión logística. Para el análisis de la supervivencia, Kaplan-Meyer, log-rank y modelos de Cox.

\section{RESULTADOS}

Analizando el valor del Gleason clínico 8-10 como prueba diagnóstica, obtenemos que el Gleason clínico 8-10 califica correctamente al 66\% de los pacientes cuando se compara con el Gleason patológico, infra-califica al 14\% de los pacientes y sobrecalifica al 34\% de los pacientes. Como prueba diagnóstica presenta una sensibilidad del 44\%(IC al 95\%:36,2\%-52\%) y una especificidad 94\% (IC al 95\%:91,6\%-95,6\%) respectivamente.

Analizando las características del grupo y comparándolo con el resto (Gleason 2-7) observamos que el grupo tiene un significativo peor PSA $(\mathrm{p}=0,01)$, peor estadio clínico $(\mathrm{p}<0,0001)$, peor estadio patológico (pT3a $(\mathrm{p}<0,0001)$ y pT3b $(\mathrm{p}<0,0001)$ ), más márgenes $(\mathrm{p}<0,0001)$ y mayor progresión bioquímica ( $\mathrm{p}<0,0001)$ (Tabla 1$)$.

Tabla 1. Características grupo Gleason clínico 8-10 vs 2-7

\begin{tabular}{lccc}
\hline Gleason & $\mathbf{2 - 7}$ & $\mathbf{8 - 1 0}$ & Valor de p \\
\hline PSA & $\begin{array}{l}\text { Media 13,48 } \\
\text { Mediana } 9,8\end{array}$ & $\begin{array}{c}16,89 \mathrm{ng} / \mathrm{ml} \\
12 \mathrm{ng} / \mathrm{ml}\end{array}$ & $<0,01$ \\
& $55 \%$ & $50 \%$ & $<0,0001$ \\
T1c & $45 \%$ & $50 \%$ & \\
>T1c & $69 \%$ & $44 \%$ & $<0,0001$ \\
$<$ PT3b & $30 \%$ & $56 \%$ & \\
pT3b & $67,2 \%$ & $48 \%$ & $<0,0001$ \\
Margen- & $32,8 \%$ & $52 \%$ & \\
Margen+ & $74,3 \%$ & $52,5 \%$ & $<0,0001$ \\
Prog BQ- & $25,7 \%$ & $47,5 \%$ & \\
Prog BQ+ & & & \\
\hline
\end{tabular}

El estado actual de los pacientes es el siguiente $62,7 \%$ (490/781) está vivo y libre de progresión bioquímica, 24,8\% (194/781) vivo en progresión bioquímica, 2,9\% (23/781) muertos por cáncer y un $1,9 \%(15 / 781)$ muertos por otra causa y un $7,6 \%$ $(59 / 781)$ perdidos.

En el estudio de progresión bioquímica de la totalidad de la serie (781 pacientes) El Gleason clínico 8-10 es un factor influyente en el estudio univariado (OR:2,61 IC 95\%: 1,7-4).

En el estudio de supervivencia libre de progresión (SLP) de la totalidad de la serie (781 pacientes) la SLP de los Gleason clínico 8-10 a 3 y 5 años es de $56 \pm 5 \%$ y $35 \pm 7 \%$, significativamente peor que la del resto del grupo $(\mathrm{p}<0,0001)$ (Fig. 1). En el estudio multivariado de los factores influyentes en la SLP 


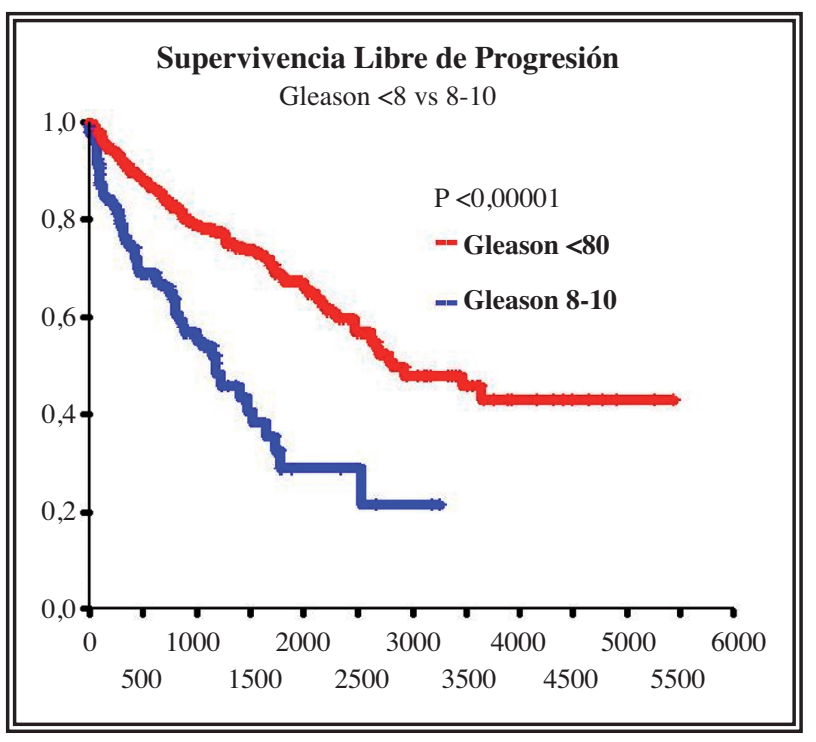

FIGURA 1. Supervivencia libre de progresión Grupo Gleason $>8$ vs 8-10.

incluye el Gleason 8-10 como factor de influencia independiente (OR:2,6 IC 95\%: 1,6-4,12) $\mathrm{p}=0,003$, junto al estadio clínico (OR:1,81 IC95\%:1,18-2,78) p<0,006, el PSA (OR: 1,03 IC 95\%:1,025-1,046) $\mathrm{p}<0,0001$ y el lado de afectación de la biopsia (unio bilateral) (OR:1,5 IC 95\%:1,01-2,24) p=0,045 (Tabla 2).

Tabla 2. Factores de influencia independiente en la supervivencia libre de progresión bioquímica, en el global de la serie.

\begin{tabular}{|c|c|c|c|}
\hline & Odds Radio & IC $95 \%$ & Valor de $p$ \\
\hline $\begin{array}{l}\text { Gleason clínico } \\
8-10\end{array}$ & 2,6 & $1,6-4,12$ & 0,003 \\
\hline Estado clínico & 1,81 & $1,18-2,78$ & 0,006 \\
\hline PSA & 1,03 & $1,025-1,046$ & $<0,0001$ \\
\hline Lado biopsia & 1,5 & $1,01-2,24$ & 0,045 \\
\hline
\end{tabular}

Dentro del Grupo Gleason Clínico 8-10 los factores influyentes en la SLP son: el PSA (OR:1,02 IC 95\%:1,003-1,04), y el estadio patológico (OR:3,84 IC 95\%:1,77-8,27) (Tabla 3). Los pacientes pT2 tienen una supervivencia significativamente mejor que los pT3 a 3 y 5 años $(80 \pm 6 \% ; 54 \pm 13 \%$ y $40 \pm 7 \% ; 27 \pm 7 \%)$ ( $<<0,0001)$. El mejor punto de corte para PSA es 11 $\mathrm{ng} / \mathrm{ml}$. Los pacientes con PSA $<1 \mathrm{lng} / \mathrm{ml}$ tienen una supervivencia a 3 y 5 años significativamente mejor que los $>11 \mathrm{ng} / \mathrm{ml}(74 \pm 7 \%, 30 \pm 22 \%$ y $40 \pm 7 \%, 26 \pm 7 \%)$ (p<0,0001) (Tabla 4 y Figs. 2 y 3).
Tabla 3. Factores de influencia en la Supervivencia Libre de Progresión Grupo Gleason 8-10

\begin{tabular}{lccc}
\hline & Odds Ratio & $\mathbf{9 5 \%}$ IC & Valor de p \\
\hline PSA $\left.{ }^{*}\right)$ & 1,02 & $1,003-1,04$ & $<0,0001$ \\
PT3 & 3,84 & $1,77-8,27$ & $<0,0001$ \\
\hline
\end{tabular}

(*) El mejor punto de corte para el PSA es $11 \mathrm{ng} / \mathrm{ml}$

Tabla 4. Supervivencia Libre de Progresión en el Grupo Gleason 8-10 a 3 y 5 años, estratificada por factores de influencia.

\begin{tabular}{lccc}
\hline & $\mathbf{3}$ años & $\mathbf{5}$ años & Valor de $\mathbf{p}$ \\
\hline PSA $<11 \mathrm{ng} / \mathrm{ml}$ & $74 \pm 7 \%$ & $30 \pm 22 \%$ & $<0,0001$ \\
$\mathrm{PSA}>1 \mathrm{lng} / \mathrm{ml}$ & $40 \pm 7 \%$ & $26 \pm 7 \%$ & $<0,0001$ \\
PT2 & $80 \pm 6 \%$ & $54 \pm 13 \%$ & $<0,0001$ \\
PT3 & $40 \pm 7 \%$ & $27 \pm 7 \%$ & $<0,0001$ \\
\hline
\end{tabular}

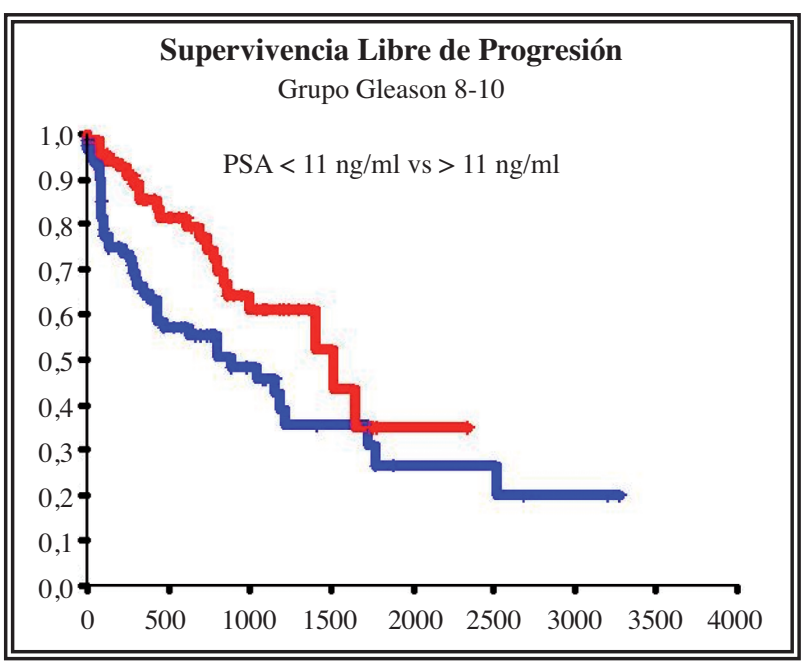

FIGURA 2. Curva de supervivencia libre de progresión bioquimica grupo Gleason 8-10, PSA<11 ng/ml vs PSA $>11 \mathrm{ng} / \mathrm{ml}$.

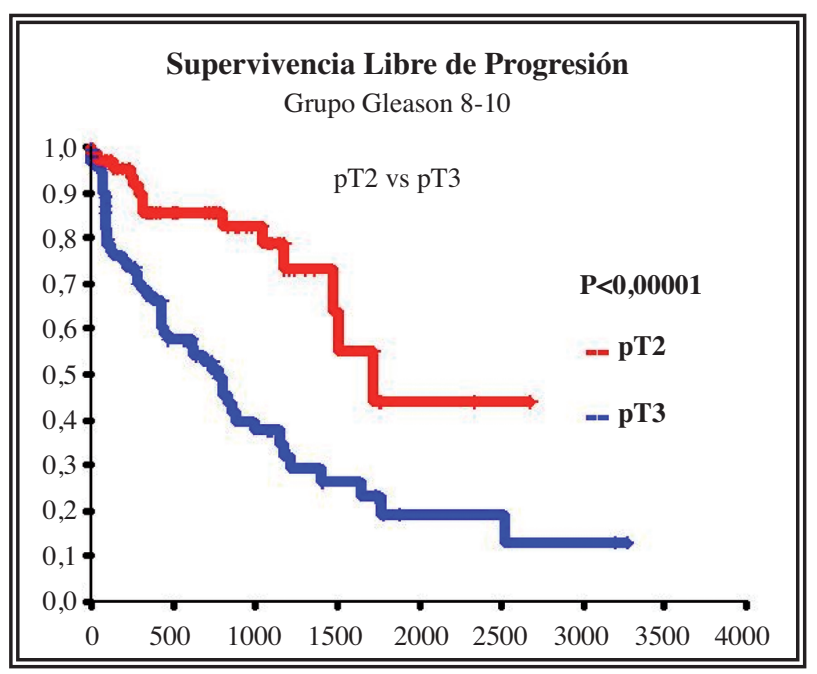

FIGURA 3. Curva de supervivencia libre de progresión bioquímica grupo Gleason 8-10, pT2 vs pT3. 


\section{DISCUSIÓN}

Los pacientes de cáncer de próstata de bajo riesgo, presentan muchas posibilidades de permanecer libres de progresión bioquímica después de prostatectomía radical, radioterapia externa o braquiterapia $^{26}$. Sin embargo, los pacientes con cáncer de próstata con Gleason clínico 8-10 presentan una mayor probabilidad de recurrencia. En la era del PSA, muchos de estos pacientes se han diagnosticado de una manera más precoz, y por tanto un potencial tratamiento curativo local es aplicable con éxito.

La incidencia actual de cáncer de próstata Gleason 8-10 en pacientes sometidos a prostatectomía radical ha descendido a un 6-10\% en la actualidad en comparación con el 16\% de la década de los ochenta. Sin embargo la proporción de pacientes con estas características y que presentaban órganoconfinación ha aumentado de un $19 \%$ a un $37 \%$ entre 1987 y 1996. La pregunta que surge es si un paciente con Gleason clínico 8-10 debe ser sometido a prostatectomía radical o no.

La información pronostica derivada de la pieza de la prostatectomía radical, es una información no disponible en el momento de la consulta inicial a la hora de decidir la opción terapéutica. Además el grado de Gleason de la biopsia no siempre es idéntico al obtenido en la pieza quirúrgica, puesto que la biopsia representa sólo una pequeña porción de la próstata. En nuestro caso la utilidad diagnóstica del Gleason clínico 8-10 tiene una sensibilidad del 44\% y una especificidad del 94\%, indicadores aceptables para modificar la actitud terapéutica a tomar.

La supervivencia libre de progresión bioquímica, así como la supervivencia libre de enfermedad en pacientes sometidos a prostatectomía radical con estadio clínico T1-2 y un Gleason clínico 8-10, no está del todo bien establecida.

Mian et al. ${ }^{27}$ estudio un grupo de 188 pacientes "limpio", sin haber recibido tratamiento neoadyuvante ni adyuvante. La supervivencia libre de enfermedad a 5 y 7 años era de $71 \%$ y del 55\% respectivamente. En el estudio multivariante la única variante de influencia independiente en la supervivencia libre de progresión bioquímica es el estadio patológico. Esta información obtenida de la pieza de prostatectomía, es una información no disponible para el clínico a la hora de tomar la decisión terapéutica en los pacientes diagnosticados de novo.
En un análisis similar, Lau et al. ${ }^{10}$ publicaron unos resultados de órgano-confinación de $26 \%$ y un porcentaje de ganglios positivos del $27 \%$, con una supervivencia global, libre de progresión y cáncer específica a 10 años de 67\%, 36\% y 85\% respectivamente. En nuestra serie el porcentaje de órganoconfinación de aquellos pacientes que presentaban Gleason clínico 8-10 fue del 44,6\%. Por el contrario, en la era pre-PSA Ohori et al. ${ }^{28}$ y Partin et al. ${ }^{29}$, aportaron unos resultados peores de órgano-confinación $8 \%$ y $12 \%$ respectivamente y peores tasas de supervivencia, por el contrario estudios recientes aportan mejores resultados de órgano-confinación entre $20 \%{ }^{30}$ y el $30 \%{ }^{9,31}$, similares a los obtenidos en el presente trabajo.

Recientemente Soloway et al. ${ }^{30}$ han publicado unos resultados de supervivencia libre de enfermedad del 59\% en aquellos que tenían un Gleason patológico de $8-10$, frente a un $68 \%$ en aquellos con Gleason patológico menor de 8 . Uno de los datos más llamativos de este estudio fue la baja incidencia de ganglios patológicos, tan solo $2,5 \%$, realizando una linfadenectomía ilio-obturatriz estándar. En el estudio univariado resultaban variables significativas para la progresión bioquímica, la presencia de margen quirúrgico positivo, extensión extrapróstatica, invasión de vesículas seminales, un volumen tumoral mayor del $20 \%$ y un PSA $\geq 20 \mathrm{ng} / \mathrm{ml}$. Sin embargo y confirmando las observaciones de Lau, obtiene que la presencia de márgenes no es una predictor de recurrencia en el estudio multivariado.

Bastian et al. ${ }^{32}$ en un estudio en el que participaban el John Hopkins (JH) y la base de datos SEARCH, presentaron un total de $21 \%$ y $41 \%$ respectivamente de órgano-confinación y márgenes negativos, en el estudio multivariado la única variable de influencia independiente en la supervivencia fue el PSA. Presentando unas supervivencias libres de progresión bioquímica del $40 \%$ y $27 \%$ a 5 y 10 años respectivamente para los pacientes del $\mathrm{JH}$ y del $32 \%$ y $28 \%$ para los del SEARCH. En aquellos pacientes con características favorables, las supervivencias libre de progresión a 5 y 10 años estimadas eran de $79 \%$ y $50 \%$ a 5 y 10 años en el grupo de $\mathrm{JH}$ y de 49\% y 49\% en la cohorte de SEARCH ${ }^{32}$.

Por su parte, el grupo de la Cleveland Clinic, encuentran órgano-confinación en un 11,7\%, extensión extracapsular en un 56,9\%, invasión de las seminales en $23,1 \%$ y ganglios patológicos en 8,9\%, 
una tasa de complicaciones del 9,7\% comparada con un $6,7 \%$ del total de pacientes sometidos a Prostatectomía radical. Con una mediana de seguimiento de 34 meses, 198 (70,4\%) se encontraban libres de progresión bioquímica ${ }^{33}$.

Nosotros obtenemos, en el estudio de supervivencia libre de progresión del total de la serie, que el Gleason 8-10, el estadio patológico, así como el lado de afectación en la biopsia son variables de influencia independiente en el estudio multivariado. Mientras que en el grupo de pacientes con Gleason 8-10 los factores de influencia en la supervivencia libre de progresión son el PSA y el estadio patológico. Obteniendo una supervivencia libre de progresión bioquímica a 4 años del 40\%.

Para aquellos pacientes con cáncer de riesgo bajo-intermedio (GS $\leq 7$ ) la prostatectomía radical es un tratamiento aceptado con unos excelentes resul$\operatorname{tados}^{30}$. Grossfeld et al. ${ }^{5}$ en una revisión de 1313 pacientes sometidos a prostatectomía radical por cáncer de próstata, observó que en aquellos pacientes caracterizados como Gleason 8-10 en la biopsia, un $38 \%$ eran clasificados como Gleason $\leq 7$ en la pieza de prostatectomía, resultados similares a los obtenidos por Manoharan ${ }^{30}$, donde este fenómeno se da en un $31 \%$ de los pacientes, quien observa a su vez una progresión bioquímica menor (32\% vs $41 \%$ ) y una menor incidencia de factores pronósticos negativos como extensión extraprostática, márgenes positivos, invasión de vesícula seminales. En nuestra serie el Gleason clínico (de la Biopsia) 8-10 tiene, como herramienta diagnóstica, una sensibilidad del 44\% y una especificidad del 94\%. Esto significa que un tercio de los pacientes catalogados como Gleason 8-10 en la biopsia realmente tiene un Gleason $\leq 7$, y por ello pueden ser rechazados para prostatectomía radical por algunos urólogos. El mismo fenómeno ocurre al revés, es decir, pacientes con un Gleason clínico $\leq 7$, que presenten un Gleason 8-10 en la pieza de prostatectomía, pero con la diferencia que su supervivencia libre de progresión es independiente del Gleason de la biopsia.

El tratamiento con radioterapia externa para un Gleason 8-10 se asocia a un 25-30\% de supervivencia libre de progresión bioquímica a 5 años ${ }^{34}$; Kupelian et al. ${ }^{35}$ publicaron una supervivencia libre de progresión bioquímica a cinco años del 47\% comparada con un $42 \%$ en aquellos sometidos a prostatectomía radical, pero con una incidencia a 8 años de recurrencia local y de metástasis superior en el grupo de radioterapia que en el de cirugía. Los resultados iniciales de la radioterapia pueden estar justificados por las diferencias de criterios a la hora de definir el fracaso bioquímico entre ambos tratamientos.

El papel de la radioterapia adyuvante sigue siendo controvertido, Do et al. $^{36}$ en una serie de 156 pacientes con cáncer de próstata de alto grado, presentó una supervivencia libre de progresión a 5 años del $65 \%$ en aquellos pacientes sometidos a radioterapia adyuvante, comparado con un 30\% en aquellos sometidos únicamente a prostatectomía radical y $25 \%$ en aquellos con radioterapia exclusivamente. Otros autores apuntaron que la radioterapia adyuvante se asocia con un menor riesgo de recurrencia bioquímica inicialmente, pero que no hay una mejora de la supervivencia cáncer especifica a 10 años ${ }^{10,16}$.

Existen trabajos comparando los resultados terapéuticos en este tipo de pacientes comparando las modalidades terapéuticas, radioterapia y cirugía ${ }^{35}$, presentando mejores resultado para los pacientes de alto grado la cirugía que la radioterapia externa, cuando se obtienen márgenes negativos.

Uno de los puntos más importantes a la hora de tratar este tipo de pacientes, es que puntos de corte utilizar a la hora de la indicación de un tratamiento $\mathrm{u}$ otro. Un estudio sobre 168 pacientes con Gleason 8-10 en la pieza de biopsia, con un seguimiento medio de 18 meses, observaron que aquellos pacientes que presentaban un PSA menor de $10 \mathrm{ng} / \mathrm{ml}$ y un porcentaje de afectación de la biopsia menor del 50\% tenían una supervivencia actuarial libre de progresión a 5 años del 67\% frente al 23\% del resto de pacientes $(\mathrm{p}=0,0001)$, por lo que recomiendan la utilización de estas dos variables a la hora de decidir que tipo de tratamiento aplicar a cada pacien$\mathrm{te}^{37}$. En nuestro caso el mejor punto de corte para PSA es de $11 \mathrm{ng} / \mathrm{ml}$, existiendo diferencias estadísticamente significativas entre ambos grupos en la supervivencia libre de progresión.

\section{CONCLUSIONES}

El Gleason Clínico 8-10 es un factor pronóstico independiente en la supervivencia libre de progresión bioquímica. Con una adecuada selección de los pacientes, PSA y estadio clínico, la prostatectomía radical continúa siendo uno de los tratamientos más válidos y con mejores resultados. 


\section{REFERENCIAS}

1. Wingo PA, Tong T, Bolden S. Cancer statistics, 1995. CA Cancer J Clin. 1995;45(1):8-30.

2. Adolfsson J, Steineck G, Whitmore WF Jr. Recent results on management of palpable clinically localized prostate cancer. Cancer. 1993;72(2):310-322.

3. Zincke H, Bergstralh EJ, Blute ML, Myers RP, Barrett DM, Lieber MM, et al. Radical prostatectomy for clinically localized prostate cancer: long-term results of 1,143 patients from a single institution. $J$ Clin Oncol. 1994;12(11):2254-2263.

4. Lu-Yao GL, McLerran D, Wasson J, Wennberg JE. An assessment of radical prostatectomy. Time trends, geographic variation, and outcomes. JAMA. 1993;269(20):2633-2636

5. Grossfeld GD, Latini DM, Lubeck DP, Broering JM, Li YP, Mehta SS et al. Predicting disease recurrence in intermediate and high-risk patients undergoing radical prostatectomy using percent positive biopsies: results from CaPSURE. Urology. 2002;59(4):560-565.

6. D'Amico AV, Whittington R, Malkowicz SB, Weinstein M, Tomaszewski JE, Schultz D, et al. Predicting prostate specific antigen outcome preoperatively in the prostate antigen era. J Urol. 2001;166 (6):2185-2188

7. Paulson DF, Piserchia PV, Gardner W. Predictors of lymphatic spread in prostatic adenocarcinoma: uro-oncology research group study. J Urol. 1980;123(5):697-699.

8. Sogani PC, Israel A, Lieberman PH, Lesser ML, Whitmore WF Jr. Gleason grading of prostate cancer: a predictor of survival. Urology. 1985;25(3):223-227.

9. Perrotti M, Rabbani F, Russo P, Solomon MC, Fair WR. Early prostate cancer detection and potential for surgical cure in men with poorly differentiated tumors. Urology. 1998;52(1):106-110.

10. Lau WK, Bergstralh EJ, Blute ML, Slezak JM, Zincke H. Radical prostatectomy for pathological Gleason 8 or greater prostate cancer: influence of concomitant pathological variables. J Urol. 2002;167(1): 117-122.

11. Borque A, Sanz G, Allepuz C, Plaza L, Gil P, Rioja LA. The use of neural networks and logistic regression analysis for predicting pathological stage in men undergoing radical prostatectomy: a population based study. J Urol. 2001;166(5):1672-1678.

12. Kattan MW, Wheeler TM, Scardino PT. Postoperative nomogram for disease recurrence after radical prostatectomy for prostate cancer. J Clin Oncol. 1999;17(5):1499-1507.

13. Partin AW, Yoo J, Carter HB, Pearson JD, Chan DW, Epstein JI, et al. The use of prostate specific antigen, clinical stage and Gleason score to predict pathological stage in men with localized prostate cancer. J Urol. 1993;150(1):110-114.

14. D’Amico AV, Whittington R, Malkowicz SB, Fondurulia J, Chen MH, Kaplan I, et al. Pretreatment nomogram for prostate-specific antigen recurrence after radical prostatectomy or external-beam radiation therapy for clinically localized prostate cancer. J Clin Oncol. 1999; 17(1): 168-172.

15. Allepuz Losa C, Borque Fernando A, Rioja Sanz LA. ¿Cuál es el algoritmo diagnóstico más apropiado para el cribado del cáncer de prós tata?. Actas Urol Esp. 2005;29(5):465-472.

16. Oefelein MG, Grayhack JT, McVary KT. Survival after radical retropubic prostatectomy of men with clinically localized high grade carcinoma of the prostate. Cancer. 1995;76(12):2535-2542.

17. Gleason DF. Classification of prostate carcinomas. Cancer Chemother Report. 1966;50(3):125-128.

18. Catalona WJ, Smith DS. 5-year tumor recurrence rates after anatomical radical retropubic prostatectomy for prostate cancer. J Urol. 1994;152(5 Pt 2):1837-1842.

19. Zincke H, Oesterling JE, Blute ML, Bergstralh EJ, Myers RP, Barrett DM. Long-term (15 years) results after radical prostatectomy for clinically localized (stage T2c or lower) prostate cancer. J Urol. 1994; 152(5 Pt 2):1850-1857.

20. Walsh PC, Partin AW, Epstein JI. Cancer control and quality of life following anatomical radical retropubic prostatectomy: results at 10 years. J Urol. 1994;152(5 Pt 2):1831-1836.
21. Grossfeld GD, Chang JJ, Broering JM, Li YP, Lubeck DP, Flanders $\mathrm{SC}$, et al. Under staging and under grading in a contemporary series of patients undergoing radical prostatectomy: results from the Cancer of the Prostate Strategic Urologic Research Endeavor database. J Urol. 2001;165(3):851-856.

22. Walsh PC. Anatomic radical prostatectomy: evolution of the surgical technique. J Urol. 1998;160(6 Pt 2):2418-2424.

23. True LD. Surgical pathology examination for the prostate gland practice survey by the American Society of Clinical Pathologist. Am J Clin Pathol. 1994;102(5):572-579.

24. Carvalhal GF, Humphrey PA, Thorson P, Yan Y, Ramos CG, Catalona WJ. Visual estimate of the percentage of carcinoma is an independent predictor of prostate carcinoma recurrence after radical prostatectomy. Cancer. 200015;89(6):1308-1314.

25. Amling CL, Bergstralh EJ, Blute ML, Slezak JM, Zincke H.. Defining prostate specific antigen progression after radical prostatectomy: what is the most appropriate cut point?. J Urol. 2001;165(4):1146-1151.

26. Grossfeld GD, Latini DM, Lubeck DP, Mehta SS, Carroll PR Predicting recurrence after radical prostatectomy for patients with high risk prostate cancer. J Urol. 2003;169(1):157-163.

27. Mian BM, Troncoso P, Okihara K, Bhadkamkar V, Johnston D, Reyes AO. Outcome of patients with Gleason score 8 or higher prostate cancer following radical prostatectomy alone. J Urol 2002; 167(4): 1675-1680.

28. Ohori M, Goad JR, Wheeler TM, Eastham JA, Thompson TC, Scardino PT. Can radical prostatectomy alter the progression of poorly differen tiated prostate cancer?. J Urol. 1994;152(5 Pt 2): 1843-1849.

29. Partin AW, Lee BR, Carmichael M, Walsh PC, Epstein JI. Radica prostatectomy for high grade disease: a reevaluation 1994. J Urol. 1994;151(6): 1583-1586.

30. Manoharan M, Bird VG, Kim SS, Civantos F, Soloway MS. Outcome after radical prostatectomy with a pretreatment prostate biopsy Gleason score of $\geq 8$. BJU Int. 2003;92(6):539-544

31. Tefilli MV, Gheiler EL, Tiguert R, Banerjee M, Sakr W, Grignon D, et al. Role of radical prostatectomy in patients with prostate cancer of high Gleason score. Prostate. 1999 Apr 1;39(1):60-66.

32. Bastian PJ, Gonzalgo ML, Aronson WJ, Terris MK, Kane CJ, Amling $\mathrm{CL}$, et al.: Clinical and pathologic outcome after radical prostatectomy for prostate cancer patients with preoperativee Gleason sum of 8 to 10. Cancer. 2006;107(6):1265-1272.

33. Berglund RK, Jones JS, Ulchaker JC, Fergany A, Gill I, Kaouk J, et al.: Radical prostatectomy as primary treatment modality for locally advanced prostate cancer: a prospective analysis. Urology. 2006;67 (6): 1253-1256.

34. Zagars GK, Ayala AG, von Eschenbach AC, Pollack A. The prognostic importance of Gleason grade in prostatic adenocarcinoma: a long-term follow-up study of 648 patients treated with radiation therapy. Int J Radiat Oncol Biol Phys. 1995;31(2):237-245

35. Kupelian P, Katcher J, Levin H, Zippe C, Suh J, Macklis R, et al External beam radiotherapy versus radical prostatectomy for clinical stage T1-2 prostate cancer: therapeutic implications of stratifi cation by pretreatment PSA levels and biopsy Gleason scores. Cancer J Sci Am. 1997;3(2):78-87.

36. Do TM, Parker RG, Smith RB, Kagan AR. High-grade carcinoma of the prostate: a comparison of current local therapies. Urology. 2001;57(6): 1121-1126.

37. Hurwitz MD, Schultz D, Richie JP, Wein AJ, Whittington R, Malkowicz SB, et al. Radical Prostatectomy for high-grade prostate cancer. Urology. 2006;68(2):367-370

Correspondencia autor: Dr. J. Rioja Zuazu

Departamento de Urología, Clínica Universitaria.

Universidad de Navarra

Av Pio XII 36 - 31008 Pamplona. Tel.: +34948255400

E-mail autor: jriojazu@gmail.com

Información artículo: Original - Cáncer de próstata

Trabajo recibido: febrero 2008

Trabajo aceptado: marzo 2008 